\title{
PCB Spiral Winding Transformer Design
}

\author{
LAI Yao-kang ${ }^{1, a,{ }^{*}}$ LI Guo-lin ${ }^{1, b}$ WU Wei-gang ${ }^{2, a}$ \\ ${ }^{1}$ Tsinghua University, HaiDian, Beijing, China \\ ${ }^{2}$ Beijing Keytone Electronic relay corporation limited, ShiJingshan, Beijing, China \\ akeytoneLL@163.com, ${ }^{\mathrm{b}}$ guolinli@tsinghua.edu.cn \\ *Corresponding author
}

Keywords : PCB, Spiral windings, Transformer, Couple.

\begin{abstract}
The present article puts forward a mathematical model for spiral windings transformer based on the PCB technique and the coils is equivalent to the concentric rings. Formulas for calculating the mutual inductance between two parallel rings are derived from Neumann formula and three simplified formulas are obtained for three special circumstances, i.e. two coils are coaxial but in different planes, in the same plain and concentric, and complete coincidence. Then the self and mutual inductances of the spiral windings are calculated. Base on theoretical calculation, the spiral winding specifications are analyzed including turns of windings, the distance between turns and between layers, the number of layers, the relationship of those between inductances, coupling coefficient. The key points relating spiral transformer design are summarized. Finally, the PCB spiral transformer electrical model is established based on the ideal transformer and practical samples are produced to verify the accuracy of the formulas and the electrical model. The theoretical formulas and the electrical model derived are suitable for circuit designs in practice.
\end{abstract}

\section{Introduction}

With the development of hybrid microelectronics technology, higher requirements for electronic components are put forward, including higher reliability, multiple functions, smaller size. But traditional transformers have large size, complicate assembly technique ${ }^{[1]}$, poor parameter consistency etc., which become the important limitation of the development of the electronic components using transformers.

The spiral winding transformers based on printing technology are widely concerned and at recent time relevant researches focus on micro transformer on chip ${ }^{[2,3]}$ and miniature transformers on $\mathrm{PCB}^{[4,5]}$.

The micro-transformer on silicon chip has the lower characteristic dimension (in $\mu \mathrm{m} \mathrm{scale}$ ) and lower inductance (within the range of $\mathrm{nH}$ ), . The relevant oscillator employing such transformer operates at higher frequency (above hundreds of $\mathrm{MHz}$ ) and larger input current (above tens of $\mathrm{mA}$ ) so that such transformers are applied to signal transportation including bus-mastering isolation, gate signal controllers etc.

The miniature transformer on $\mathrm{PCB}$ has larger characteristic dimension and relative higher inductance than those on chip, which is suitable for DC convertors and gate driving units. 
At the present time, the research on PCB transformers focuses more in the function realization or theoretical derivation of inductance and less in the design method, characteristics analysis and electrical model establish. Combining the theoretical derivation, formula simplification, circuit simulation, this paper gives out the design key points for the spiral winding transformer based on PCB. Also, the paper analyzes the transformer characteristics in detail and establishes an electrical model which may apply to DC convertors and gate drive circuit directly.

\section{Principle and design}

Figure 1 shows a spiral wingding transformer based on PCB, with the parameters including the inductance of primary and secondary windings $\mathrm{L}$, mutual inductance $\mathrm{M}$, winding resistance $\mathrm{R}$, parasitic capacitance $\mathrm{C}$, etc.

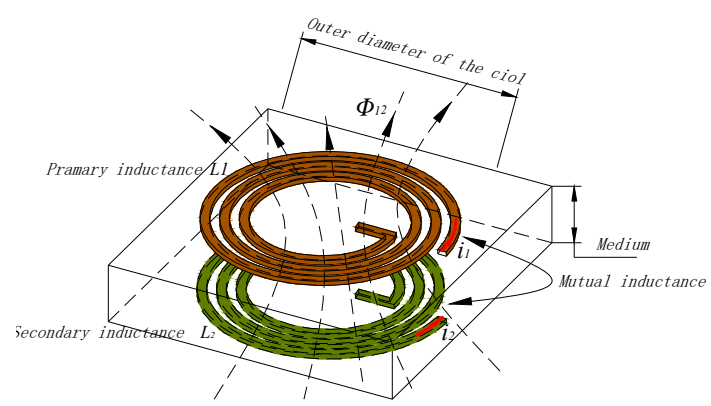

Figure 1 Spiral windings transformer

\subsection{Equivalent circle for spiral winding}

A spiral winding with $\mathrm{n}$ turns is shown in Figure 2a):
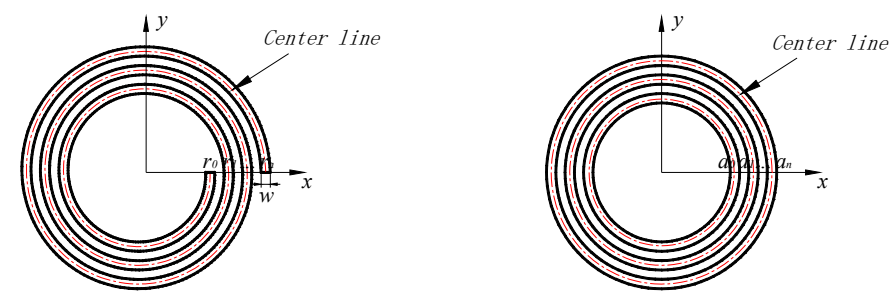

a) spiral winding with $n$ turns $b$ ) $n$ concentric rings

Figure 2 Equivalent windings for $\mathrm{n}$ turns spire

The width of the coil track is $\mathrm{w}$, its center line beginning from the inner point with radius $\mathrm{r}_{0}$, one turn is according to one circle, the end turn with the radius $r_{n}$. The mathematic expression is

$$
\left\{\begin{array}{l}
x=\left(r_{i-1}+\frac{\theta}{2 \pi} \Delta r\right) \cos \theta \\
y=\left(r_{n-1}+\frac{\theta}{2 \pi} \Delta r\right) \sin \theta \quad \theta \in(0,2 \pi), i=1,2 \ldots, n(1) \\
r_{i}-r_{i-1}=\Delta r
\end{array}\right.
$$

Obviously, when $\Delta \mathrm{r}$ is small enough, the $\mathrm{n}$ turns of spiral winding can be expressed as the concentric circles, as shown in Figure $2 b$ ), The radius of circle is $a_{i}=\frac{r_{i}+r_{i-1}}{2}$.

\subsection{Calculation of mutual and self-inductance}

For any closed current path in space, the inference of Neumann formula ${ }^{[6]}$ can be used to calculate the mutual and self-inductance between the two conductors when the path is practical conductors. 

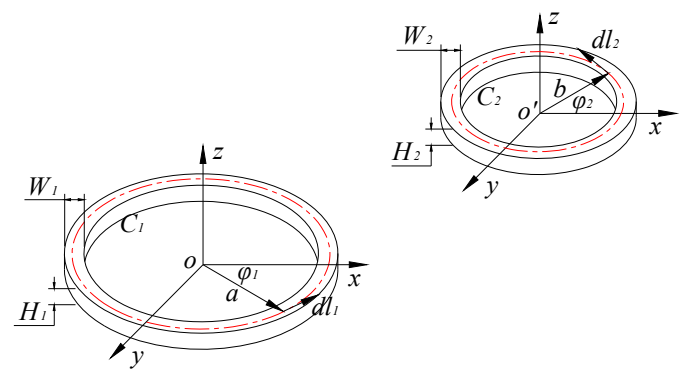

Figure 3 Paralleled cirque conductors

Figure 3 shows two paralleled circle conductors, $\mathrm{C}_{1}$ and $\mathrm{C}_{2}$. Take the center coordinate of $\mathrm{C}_{1}$ as o $(0,0,0)$, radius as a, circle width of conductor as $\mathrm{W}_{1}$, circle thickness as $\mathrm{H}_{1}$. Take the center coordinate of $\mathrm{C}_{2}$ as o' ( $\mathrm{p}, \mathrm{k}, \mathrm{z}$ ), radius as $\mathrm{b}$, width of conductor as $\mathrm{W}_{2}$, thickness as $\mathrm{H}_{2}$. Convert the vector integral to angular integral, At the same time, assume the current in the coil is unified for simplicity, the expression for mutual inductance of the two circles is derived as:

$$
M_{12}=\frac{\mu_{0}}{4 \pi} \frac{1}{W_{1} H_{1} W_{2} H_{2}} \int_{a 1}^{a 2} \int_{-\frac{H_{1}}{2}}^{\frac{H_{1}}{2}} \int_{b 1}^{b 2} \int_{-\frac{H_{2}}{2}}^{\frac{H_{2}}{2}} \int_{0}^{2 \pi} \int_{0}^{2 \pi} \frac{a b \cos \left(\varphi_{1}-\varphi_{2}\right) d \varphi_{1} d \varphi_{2} d a d h_{1} d b d h_{2}}{\sqrt{\left[a \cos \varphi_{1}-\left(b \cos \varphi_{2}+p\right)\right]^{2}+\left[a \sin \varphi_{1}-\left(b \sin \varphi_{2}+k\right)\right]^{2}+\left(z+h_{1}-h_{2}\right)^{2}}}(2)
$$

Where $a_{1}$ and $a_{2}$ are the inner and outer radius of the coil $C_{1}$ respectively, $b_{1}$ and $b_{2}$ are the inner and outer radius of the coil $\mathrm{C}_{2}$ respectively.

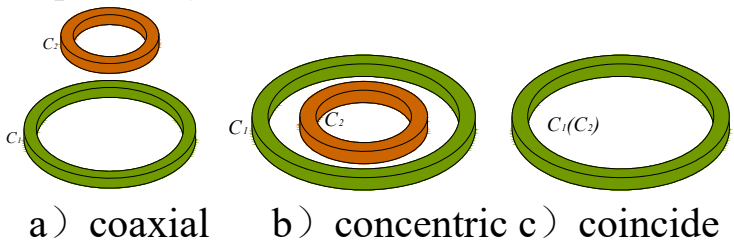

Figure 4 Three special conditions

Consider the three special conditions shown in Figure 4

a) When $\mathrm{C}_{1}$ and $\mathrm{C}_{2}$ is coaxial, the mutual expression is simplified as

$$
M_{12}=\frac{\mu_{0}}{2} \frac{1}{W_{1} H_{1} W_{2} H_{2}} \int_{a 1}^{a 2} \int_{-\frac{H_{1}}{2}}^{\frac{H_{1}}{2}} \int_{b 1}^{b 2} \int_{-\frac{H_{2}}{2}}^{\frac{H_{2}}{2}} \int_{0}^{2 \pi} \frac{a b \cos \theta d \theta d a d h_{1} d b d h_{2}}{\sqrt{\left(z+h_{1}-h_{2}\right)^{2}+a^{2}+b^{2}-2 a b \cos \theta}}
$$

b) When $\mathrm{C} 1$ and $\mathrm{C} 2$ is concentric, the mutual expression is simplified as:

$$
M_{12}=\frac{\mu_{0}}{2} \frac{1}{W^{2} H} \int_{a 1}^{a 2} \int_{-\frac{H}{2}}^{\frac{H}{2}} \int_{b 1}^{b 2} \int_{0}^{2 \pi} \frac{a b \cos \theta d \theta d a d h d b}{\sqrt{h^{2}+a^{2}+b^{2}-2 a r \cos \theta}}
$$

c) When the coil $\mathrm{C}_{1}$ and $\mathrm{C}_{2}$ coincide, the expression becomes the self-inductance calculating formula.

Then for the primary and secondary coils shown in Figure 1, the total self-inductance is:

The total mutual inductance is:

$$
L_{\text {self inductance }}=\sum_{i=1}^{n} L_{i}=\sum_{i=1}^{n} \mathrm{M}_{i i}
$$

The total inductance is:

$$
M=\sum_{i=1}^{n} \sum_{j=1, j \neq i}^{n} \mathrm{M}_{i j}
$$

$$
L=\sum_{i=1}^{n} \sum_{j=1}^{n} \mathrm{M}_{i j}
$$

The mutual inductance between primary and secondary coils can be calculated in the same way as mentioned above.

\subsection{D.C. resistance}

For a spiral winding with $\mathrm{n}$ turns, the D.C. resistance is:

$$
R=\sum_{i=1}^{n} \mathrm{R}_{i}=\frac{2 \pi \rho}{W H} \sum_{i=1}^{n} a_{i}
$$


Where $\rho$ is the resistivity of the conductor, $a_{i}$ is the equivalent radius of the $i$ turn, $W$ and $H$ represents the width and thickness of the conductor respectively.

\subsection{The parasitic capacitance of primary winding}

Only special cases are discussed: When the upper and lower windings (primary and secondary windings) are coaxial, and the two have the same size, same width, the same distance between the conductor and the sane winding direction, the parasitic capacitance between the two is:

$$
C_{12}=\varepsilon_{0} \varepsilon_{r} \frac{s}{d}=\varepsilon_{0} \varepsilon_{r} \frac{\pi\left(a_{n}^{2}-a_{1}^{2}\right)}{2 d}
$$

Where $\varepsilon_{0}$ is vacuum dielectric constant, $\varepsilon_{\mathrm{r}}$ is relative permittivity of materials, $d$ is medium thickness, $\mathrm{a}_{n}$ is the radius of the equivalent conductor of the $\mathrm{n}$ turn.

When the windings are winded in reverse direction, the overlapping area is reduced by nearly half, and then the parasitic capacitance is also reduced nearly half.

\subsection{Coil characteristics analysis}

Mathematics software is applied combining formula (3) to (9) to calculate and fit the relevant curves, the analysis obtained is applicable to general situation

\subsubsection{Characteristics of single layer coil}

Design an n-turn spiral transformer shown in Figure 5. The winding parameters are: starting radius: $r_{o}=32 \mathrm{mil}$, the distance between turns $\Delta r=8.1 \mathrm{mil}$, conductor width $W=4 \mathrm{mil}$, thickness $H=0.7 \mathrm{mil}$.

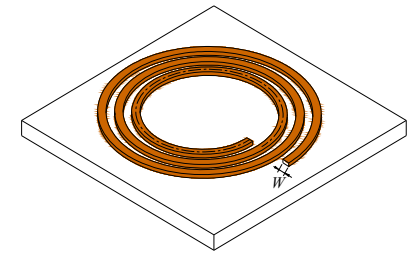

Figure 5 Windings with $\mathrm{n}$ turns

The self-inductance of turn $\mathrm{i}$ is shown in Figure 6a), the mutual inductance between turn 1 and turn $\mathrm{i}$ is shown in Figure 6b), the total inductance of the coil is shown in Figure $6 \mathrm{c}$ ), the coil Q value (calculating under $1 \mathrm{MHz}$ ) is shown in Figure 6d).

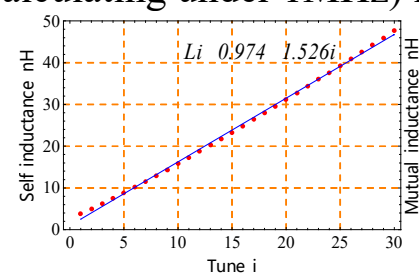

a) self-inductance

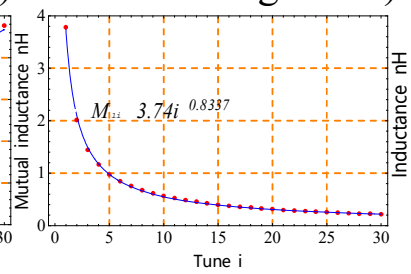

b) mutual inductance

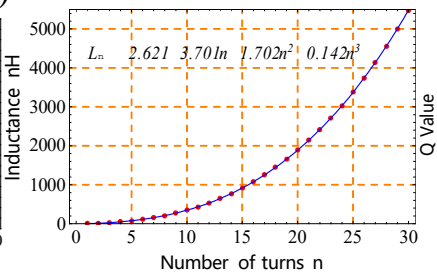

c) total inductance

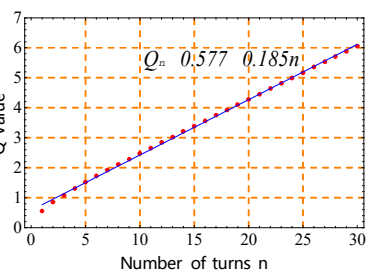

d) Q value

Figure 6 Self-inductance, mutual inductance and total inductance

Following points of summary are obtained from Figure 6:

a) The total inductance include self-inductance and mutual inductance. From turn i to turn $i+1$, the self-inductance increases by $\mathrm{L}_{\mathrm{i}+1}$ lineally. However, the mutual inductance is $\sum_{i=1}^{n} M_{i, n+1}$, increasing by cubic function. Obviously, as the number of turns increases, the mutual inductance between the turns will possess more proportion. But the mutual inductance decreases by power function as the distance between the turns increasing. Hence in order to obtain lager inductance, the 
distance between turns is to be as small as possible.

b) As the mutual inductance between coils possess a large proportion, under the restricted area, the more the number of turns (the smaller the distance between the turns), the lager is the inductance obtained.

c) Outer turns have the larger influence to the inductance. Thus the inner turns may be designed a little large to leave more room to place pads.

d) The coil Q value is lower. The cross-section area of the conductor is conditionally as large as possible.

\subsubsection{The characteristics of $\mathbf{N}$ layer stack windings}

As shown in Figure 7, an $\mathrm{N}$ layers stack structure windings with the same parameters as Figure 5 is demonstrated. The thickness of dielectric layer (distance between layers) is $d=5 \mathrm{mil}$. The adjacent coil is in reverse direction. The current is in the same annular direction and then the mutual inductance is enhanced so that the total inductance is increased.

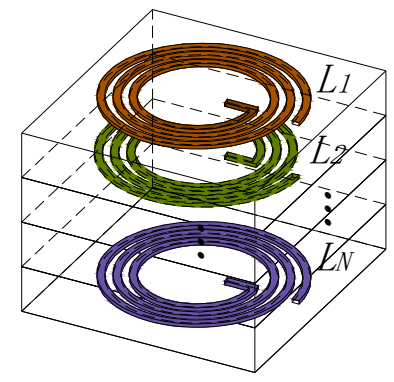

Figure 7 Windings with $\mathrm{N}$ layers

In Figure 7, when $\mathrm{L}_{1}$ and $\mathrm{L}_{2}$ have the same turns, the variation of the mutual inductance with the number of turns is shown as Figure 8a), the variation of the mutual inductance with coincident area is shown in Figure 8b), the variation of the coupling coefficient between $L_{1}$ and $L_{2}$ with number of turns is shown as Figure 8c). The variation of the coupling coefficient with the distance between the two coils is shown in Figure 8d). When $L_{1}$ keeps 10 turns, the coupling coefficient between $L_{1}$ and $\mathrm{L}_{2}$ varies with the number of turns of $\mathrm{L}_{2}$ is shown in Figure $8 \mathrm{e}$ ). When $\mathrm{L}_{1} \sim \mathrm{L}_{\mathrm{N}}$ have the same turns and shape, and the coils on all layers are so connected that the inductance is enhanced, the variation of the ratio of total inductance to inductance of single layer with the total number of layers is shown in Figure 8f). 

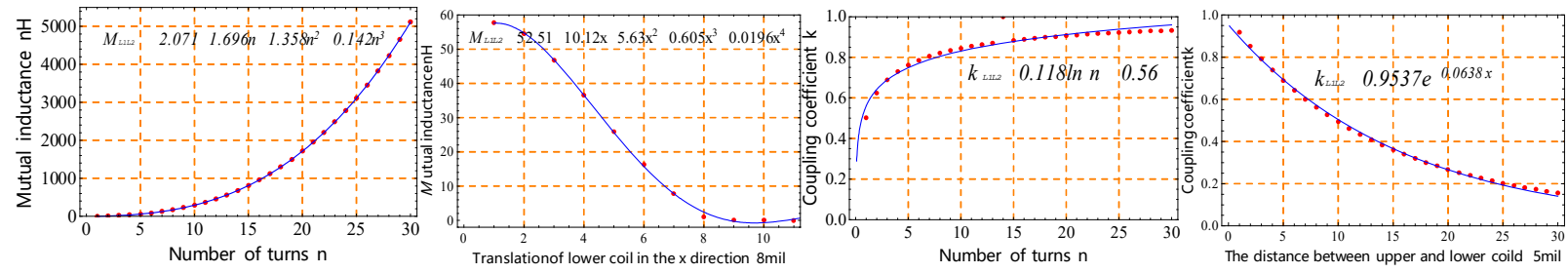

a) mutual inductance between coils b) mutual inductance vs. stack area c) coupling coefficient vs. number of turns d) coupling coefficient vs. distance

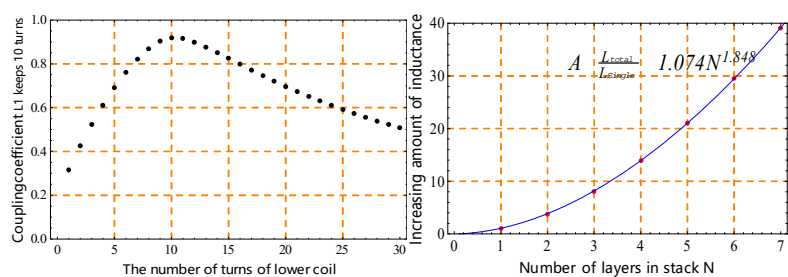

e) The coupling coefficient under the upper coil has 10 turns f) increasing amount of inductance under $\mathrm{N}$ layers in series

Figure 8 Mutual inductance, couple coefficient, increasing amount of inductance

From the curves in Figure 8, following conclusion can be used as the guidance to the designs of spiral winding transformers:

a) When all windings have the same size and number of turns, and all of them are coaxial, the maximum coupling coefficient between primary and secondary windings is obtained.

b The coupling coefficient decreases exponentially with the increasing distance between upper and lower coils. The influence is obviously so that the thickness of the dielectric layer is as little as possible during the transformer design. But this may influence parasitic capacitance and dielectric withstand voltage between primary and secondary windings.

c) The inductance of a coil increases nearly square relation with the number of stack layers so that more layers may be selected during the transformer design in order to obtain larger inductance of the windings. But the cost will increases.

\subsection{Electrical model for transformer}

Spiral winding transformer is a kind of coreless transformer which is shown in Figure 9

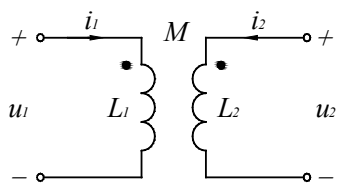

a) coreless transformer

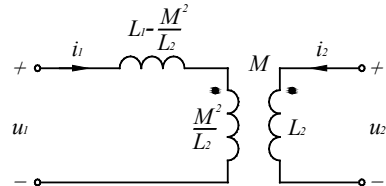

b) unity coupling transformer

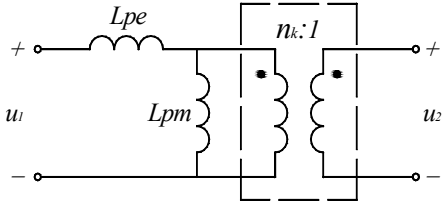

c) ideal transformer

Figure 9 Equivalent circuit of transformer

For a transformer in Figure $9 \mathrm{a}), \mathrm{L}_{1}$ is primary inductor, $\mathrm{L}_{2}$ is the secondary inductor, $\mathrm{M}$ is the mutual inductor. The couple coefficient of the transformer is $k=\frac{M}{\sqrt{L_{1} L_{2}}}<1$. Figure 9a) can be equivalent to a transformer containing ideal transformer circuit $^{[7]}$ as shown in Figure 9b).Furthermore, the ideal transformer can be equivalent to the ideal transformer with several paralleled inductors cascade and the voltage ratio is $n_{k}=\frac{M}{L_{2}}$, as shown in Figure 9c).

Taking into account the practical application, we get the complete equivalent circuit for spiral transformer as shown in Figure 10a): 


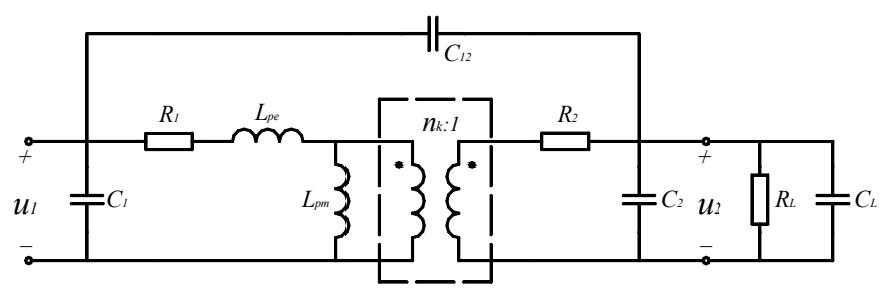

Figure10 Model for spiral windings transformer

Where leakage inductance is $L_{p e}=\left(1-k^{2}\right) L_{1}$, magnetizing inductance is $L_{p m}=k^{2} L_{1}, R_{1}$ and $R_{2}$ are the winding D.C. resistors of the primary and secondary coils, $C_{1}$ and $C_{2}$ are the parasitic capacitors of primary and secondary coils respectively, $C_{12}$ is the parasitic capacitor between primary and secondary coils, $C_{L}$ is the load capacitor, $R_{L}$ is the load resistor.

The transformer circuit shown in Figure 10 is converted into the circuit in frequency domain shown in Figure 11.

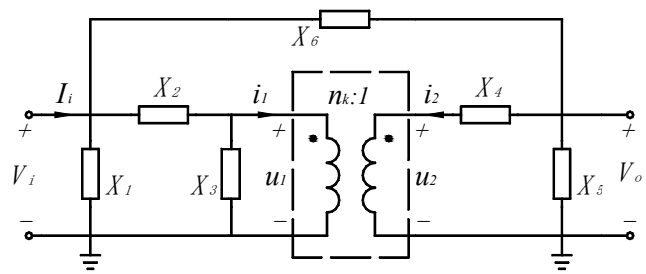

Figure11 Circuit in frequency domain

Where $X_{1}=\frac{1}{s C_{1}}, X_{2}=R_{1}+s\left(1-k^{2}\right) L_{1}, X_{3}=s k^{2} L_{1}, X_{4}=R_{2}, X_{5}=\frac{R_{L}}{s R_{L}\left(C_{2}+C_{L}\right)+1}, X_{6}=\frac{1}{s C_{12}}$.

The transfer function and input impedance are obtained.

Transfer function: $A=\frac{V_{o}}{V_{i}}=\frac{\frac{1}{X_{2}\left(\frac{1}{n_{k} X_{4}}+\frac{n_{k}}{X_{2}}+\frac{n_{k}}{X_{3}}\right)}+\frac{X_{4}}{X_{6}}}{X_{4}\left(\frac{1}{X_{4}}+\frac{1}{X_{5}}+\frac{1}{X_{6}}\right)-\frac{1}{n_{k} X_{4}\left(\frac{1}{n_{k} X_{4}}+\frac{n_{k}}{X_{2}}+\frac{n_{k}}{X_{3}}\right)}}$

Input impedance: $Z_{\text {in }}=\frac{\mathrm{V}_{i}}{I_{i}}=\frac{1}{\frac{1-\mathrm{A}}{X_{6}}+\frac{1}{X_{1}}+\frac{1}{X_{2}}-\frac{n}{X_{2} Y_{1}}\left(\frac{1}{X_{2}}+\frac{A}{n_{k} X_{4}}\right)}$

\section{Experiment}

10 layers of FR4 board is manufactured as shown in Figure 12 according to the structure and size given in 2.4 .

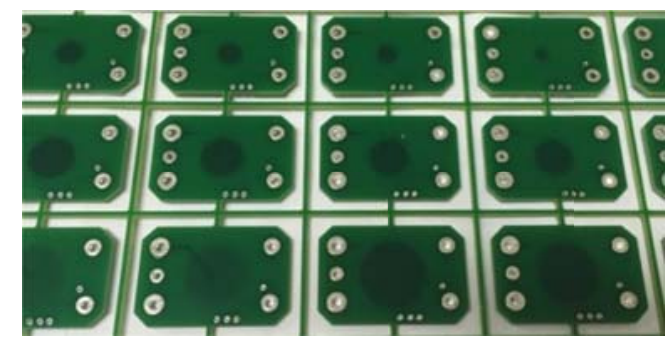

Figure 12 Various standard spiral windings

Test data is shown in table 1(equipment: Instek LCR-8101G, test condition:1MHz.0.1V). 
Table 1 Spiral winding test

\begin{tabular}{ccccc}
\hline & & & Inductance $(\mathrm{nH})$ & \\
\hline Coil structure & Coil external diameter $(\mathrm{mm})$ & Theoretical calculation & Practical measurement & deviation \\
$1 \times 6$ & 4.0 & 111.2 & 107.2 & $3.60 \%$ \\
$1 \times 10$ & 5.6 & 346.8 & 331.8 & $4.5 \%$ \\
$1 \times 20$ & 9.75 & 1889.4 & 1889.8 & $0.02 \%$ \\
$1 \times 25$ & 11.8 & 3374.5 & 3326.8 & $1.43 \%$ \\
$2 \times 6$ & -- & 406.6 & 421 & $3.54 \%$ \\
$2 \times 10$ & -- & 1279.5 & 1214 & $4.3 \%$ \\
$2 \times 20$ & -- & 7204.5 & 6893 & $4.3 \%$ \\
$2 \times 25$ & -- & 12874.3 & 12850 & $0.19 \%$ \\
$3 \times 10$ & -- & 2536.2 & 2474 & $2.5 \%$ \\
$4 \times 10$ & -- & 4501.7 & 4325 & $4.09 \%$ \\
$5 \times 10$ & -- & 6620.5 & 6468 & $2.36 \%$ \\
$6 \times 10$ & -- & 8113.6 & 7917 & $2.48 \%$ \\
$7 \times 10$ & -- & 12174 & 11690 & $4.14 \%$ \\
\hline
\end{tabular}

Where $\mathrm{A} \times \mathrm{B}$ represents $\mathrm{B}$ turns of the winding in A layer in stack structure.

According to Table 1, the deviation between the calculation and practice measurement is within $5 \%$ so that the theoretical analysis is accurate and the specification of the coill obtained is correct. It is suitable for the guidance to the design of the spiral windings and transformers.

The PCB transformer sample is shown in Figure 13a) and its internal connection is shown in Figure 13b)

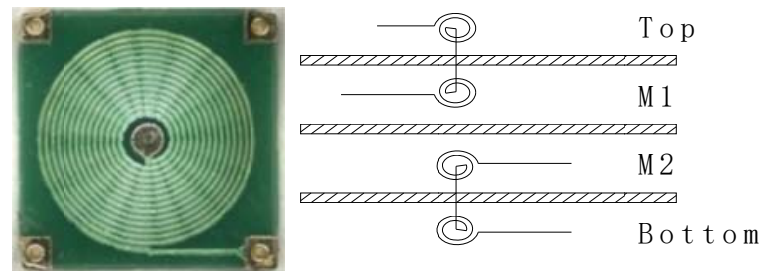

a) $2 \times 14: 2 \times 14$ transformer b ) internal structure

Figure 13 Spiral winding transformer and its internal structure

The test data for the transformer is shown in Table 2.(signal generator: Tektronix AFG3022).

Table 2 Transformer test

\begin{tabular}{lll}
\hline & $\begin{array}{l}\text { Spiral winding } \\
\text { transformer }\end{array}$ & $\begin{array}{l}\text { NXO-100core } \\
\text { transformer }\end{array}$ \\
\hline Transformer dimension $(\mathrm{mm})$ & $8.6 \times 8.6 \times 1.0$ & $\varphi 5$ magnetic core $\varphi 5$ \\
Number of board layers, distance between layers $(\mathrm{mm})$ & $4 、 0.25$ & $11: 11$ \\
Number of turns, voltage ratio & $2 \times 14: 2 \times 14$ & $3.26 、 3.12$ \\
Primary, secondary inductance $(\mu \mathrm{H})$ & $2.75,2.77$ & 0.725 \\
Coupling coefficient & 0.741 & $0.18 、 0.18$ \\
DC resistance of primary and secondarywindings $(\Omega)$ & $3.42 、 3.26$ & $<1 、<1$ \\
Parasitic capacitance of primary and secondary windings $(\mathrm{pF})$ & $<1 、<1$ & $<1$ \\
Parasitic capacitance between primary and secondary windings $(\mathrm{pF})$ & 6.4 & $<1$ \\
\hline
\end{tabular}

Applying loads to output of the transformer obtains the output voltage vs. frequency curve as shown in Figure 14a), impedance module vs. frequency curve is shown in Figure 14b). The load is composed of $\mathrm{R}_{\mathrm{L}}=100 \mathrm{k} \Omega, \mathrm{C}_{\mathrm{L}}=470 \mathrm{pF}$. 

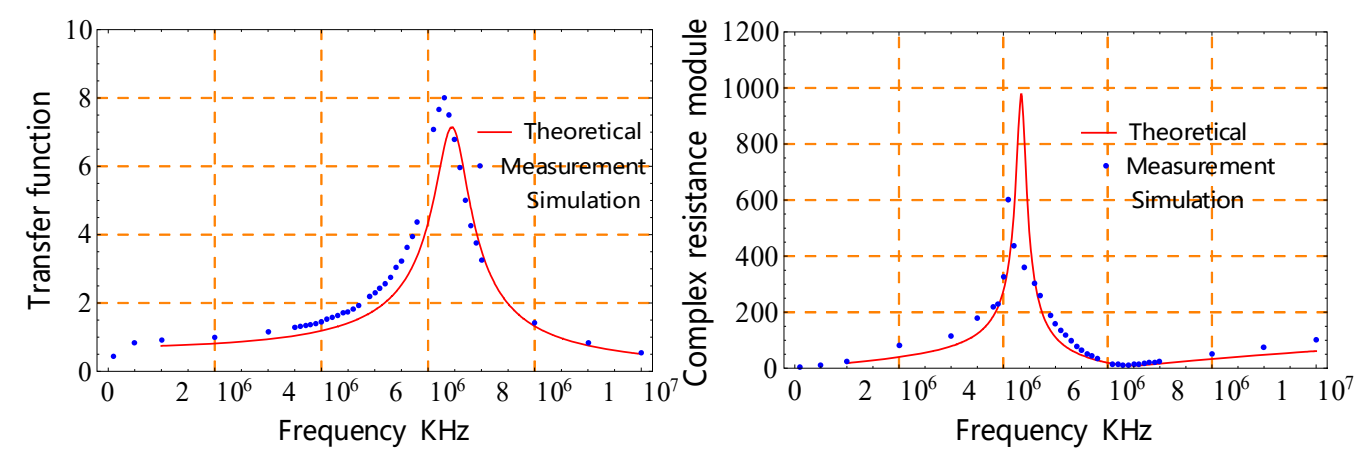

a) frequency specification of transfer function b ) frequency specification of complex resistance module

Figure 14 Spiral winding transformer' s amplitude and absolute value of resistance.

Figure 14 shows that the three curves are similar and the accuracy of the transformer model is verified.

\section{Conclusions}

The formula for spiral transformer calculation provided in this paper has the simple format, small amount of calculation and accurate result. The analysis based on the formula is satisfied, and the results could apply to common PCB spiral transformer windings calculations and transformer design. The electrical model of the transformer is accurate and may apply to DC convertor circuits design and MOSFET gate drive circuit design.

\section{References}

[1]Yuan Cong, GuoZhongning , Overview on Micro-joining Technology Using Enameled Wire[J],Electrical machining and mould,2013,22-25

[2]EL-GHARNITIO,KERHERVE E,BEGUERET J B,etal.Modeling of integrated monolithic transformers for silicon RF IC[C],IEEE IntConf Elec,Circ\&Syst.Nice,France, 2004:137-140

[3]RongxiangWu,Johnny K.O. Sin,” A Novel Silicon-Embedded Coreless Transformer forIsolated DC-DC Converter Application”, 2011 IEEE 23rd International Symposium on Power Semiconductor Devices and ICs (ISPSD), May, 2011,pp.352-355

[4] A. Majid, H.B.Kotte, J.Saleem,", High Frequency Half-Bridge Converter using Multilayered Coreless PrintedCircuit Board Step-Down Power Transformer", 2011 IEEE 8th International Conference on Power Electronics and ECCE Asia (ICPE \& ECCE), 2011,pp.1177-1181

[5]HariBabuKotte," High-Speed (MHz) Series Resonant Converter (SRC)Using Multilayered Coreless Printed Circuit Board(PCB) Step-Down Power Transformer", IEEE Transactions On Power Electronics, Vol. 28, No. 3, March 2013,pp.1253-1264

[6] Lin Weigan, Fu Guohang, electromagnetic field theory [M], Beijing people's Posts and Telecommunications Press, 1996,295-300

[7]Qiu Guanyuan. Circuit [M]. Beijing: Higher Education Press, 1985:280 